\begin{tabular}{|c|c|}
\hline Title & $\begin{array}{l}\text { Understanding formation of photonic bandgap edge for maximum propagation angle in all-solid photonic bandgap } \\
\text { fibers }\end{array}$ \\
\hline Author(s) & Murao, Tadashi; Nagao, Koy uru; Saitoh, Kunimasa; Koshiba, Masanori \\
\hline Citation & $\begin{array}{l}\text { Journal of the Optical Society of A merica B : Optical Physics, } 28(3), 453-461 \\
\text { https://doi.org/10.1364/JOSA B.28.000453 }\end{array}$ \\
\hline Issue Date & 2011-03-01 \\
\hline Doc URL & http:/hdl.handle.net/2115/45024 \\
\hline Rights & (c) 2011 Optical Society of A merica \\
\hline Type & article \\
\hline File Information & JOSAB28_3_453-461.pdf \\
\hline
\end{tabular}

Instructions for use 


\title{
Understanding formation of photonic bandgap edge for maximum propagation angle in all-solid photonic bandgap fibers
}

\author{
Tadashi Murao,* Koyuru Nagao, Kunimasa Saitoh, and Masanori Koshiba \\ Graduate School of Information Science and Technology, Hokkaido University, Sapporo 060-0814, Japan \\ ${ }^{*}$ Corresponding author: murao@icp.ist.hokudai.ac.jp
}

Received September 3, 2010; revised December 20, 2010; accepted December 21, 2010; posted December 21, 2010 (Doc. ID 134565); published February 18, 2011

\begin{abstract}
We propose a simple formula that gives the effective refractive index of the first-order photonic bandgap (PBG) edge for the maximum propagation angle, which corresponds to the PBG floor in all-solid photonic bandgap fibers (PBGFs). In particular, based on an antiresonant reflecting optical waveguide (ARROW) theory incorporating a phase relation between resonant modes in low-index regions for perpendicular direction of the light propagation, we show that the effective index of the PBG edge at a wavelength does not depend on the diameter and refractive index of high-index rods, and is largely determined only by the pitch when normalized rod diameters are small. In other words, it has a constant value for the structural parameters with which the PBG emerges at the same wavelength normalized by the pitch. Moreover, the source of rod-diameter and refractive-index dependences on the condition for the formation of the PBG edge is demonstrated when the diameter and the refractive index of high-index rods are effectively large. Finally, the validity of the proposed simple formula, which can be applied for any structural parameters, is verified with the vectorial solver based on the finite-element method (FEM). (C) 2011 Optical Society of America OCIS codes: $\quad 060.2310,060.5295,230.5298$.
\end{abstract}

\section{INTRODUCTION}

Recently, all-solid photonic bandgap fibers (PBGFs) [1-13], which are one of the types of solid-core PBGFs, have attracted considerable attention due to their unusual transmission characteristics that cannot be obtained by conventional optical fibers. In all-solid PBGFs, the cladding is composed of periodically arranged high-index rods and the core is formed by omitting one or several rods. Owing to the photonic bandgap (PBG) effect of the photonic-crystal structure for light with out-of-plane propagation, a fundamental core mode is supported in the defected core region with effectively low losses. Because of the functionality pattern of alternate transmission and transmission-inhibited bands exhibited by the photoniccrystal cladding, the PBGFs can be applied to several devices. In particular, compared to the other type of solid-core PBGFs whose high-index rods are composed of high-index liquid [14-18], because of its higher degree of compatibility with all-glass conventional fibers, the all-solid type whose cladding is composed of Ge-doped rods in silica can easily be incorporated into fiber amplifiers or fiber lasers as fiber-type optical filters.

The photonic-crystal structure in the cladding of all-solid PBGFs generates a number of PBG orders separated by photonic bands associated with linearly polarized (LP) rod modes [19], as will also be discussed in the next section. It is known that, in general, odd-order PBGs have a greater PBG depth compared to the even-order PBGs, because of their smaller modal overlap characteristic that reduces the effect of enlargement of bandwidth on the PBG edge, according to Ref. [20]. Among all of the odd-order PBGs, the third-order PBG supports a lower confinement loss property than the first-order PBG [5] because the propagation angle for the core modes is lower in the third-order PBG; in other words, the core radius relative to the wavelength is larger in most cases. However, we have recently shown that the first-order PBG exhibits a lower bending loss property than the third-order PBG [21], and the exploitation of the first-order PBG has also attracted attention in recent years, by resolving the issue of the high confinement loss property [르.

In order to understand the properties of all-solid PBGFs, an important objective has been construction of a theory for the wavelength and propagation constant (propagation angle) of light with out-of-plane propagation at which a PBG emerges [23], because the wavelength is directly related to the transmission band, and the propagation constant to the number of allowed propagation modes [24,25] and the impact of bending losses $[20,21,26]$. When the PBG depth, defined as the difference between the effective indices of silica and the PBG edge, increases, a large number of guided modes are to be supported and, on the other hand, this also leads to a tightbending structure if the effective index of the core mode is constant [27]. As for the transmission band, it can successfully be estimated by using the theory for conventional antiresonant reflecting optical waveguides (ARROWs) [28-32]. Provided that each high-index rod in the cladding is considered as a core of conventional fibers, the condition at which guided modes are localized in rods corresponds to resonance for the transverse direction, and the other condition (when the guided modes are nonlocalized) is antiresonance or PBG. Although some degree of modification of the ARROW theory is required due to the modal overlaps between the modes supported by each highindex rod [19], because of their high localization characteristic at the effective index where the fundamental core mode of the PBGFs exists (near the refractive index of silica substrate), 
the effect of the modal overlap on the wavelength shift of the transmission band is effectively small. As for the propagation constant (effective refractive index), it is known that the PBG edge for the maximum propagation angle is determined by the resonant condition in the low-index region between rods [33], as in ARROWs [29]. However, because of the low localization characteristic of the modes supported in low-index regions between high-index rods, it is difficult to estimate the PBG edge by using the ARROW theory. Recently, it has been shown that an approximate band structure can be obtained successfully by considering the condition for the even and odd rod modes applying an appropriate boundary condition [33].

In this paper, we propose a simple formula that gives the effective refractive index of the first-order PBG edge for the maximum propagation angle, which corresponds to the PBG floor in all-solid PBGFs, based on an ARROW theory incorporating a phase relation between resonant modes in lowindex regions. We show that the effective index of the PBG edge at a wavelength does not depend on the diameter and the refractive index of high-index rods, and it is comprehensively determined only by the pitch when normalized rod diameters are small. In other words, it has a constant value for the structural parameters with which the PBG emerges at the same wavelength normalized by the pitch. By incorporating the ARROW theory for high-index rods, it is theoretically shown that the PBG depth increases monotonically with the relative refractive-index difference and quadratically with the rod diameter normalized by the pitch. Moreover, the cause of rod-diameter and refractive-index dependences on the condition for the formation of the PBG edge is demonstrated when the diameter and the refractive index of high-index rods are large. It is shown that the change of the absolute value of wave vector for the resonant modes in low-index regions and the effect of modal overlap of rod modes are responsible for the factor of reduction of PBG depth. The validity of the proposed simple formula that can be applied for any structural parameters is verified by means of the vectorial solver based on the finite-element method (FEM) [34]. Finally, a critical reason for decreasing the PBG depth in even-order PBGs is discussed, based on the theory provided here.

\section{UNDERSTANDING FORMATION OF PHOTONIC BANDGAP EDGE FOR MAXIMUM PROPAGATION ANGLE}

Figure 1(a) shows a schematic representation of the light propagation out of plane into a photonic crystal, assuming the cladding of a typical all-solid PBGF. The photonic-crystal structure is composed of a periodic arrangement of high-index rods (with refractive index $n_{\text {high }}$ ) as the triangular lattice with diameter $d$ and pitch $\Lambda$ in a silica background (with refractive index $n_{\text {low }}=1.45$ ). In PBGFs, because light propagates along the longitudinal direction (out-of-plane propagation), it possesses the longitudinal component of wave vector (propagation constant). Because the wave equations for electromagnetic fields have a dependence on the propagation constant, the resultant frequencies of the PBG for the perpendicular direction of the propagation axis also depend on the propagation constant [23]. Here, we consider an incident light to the photonic crystal with the absolute value of wave vector of $k$ in the material and propagation constant of $\beta$. The transverse component of the wave vector is represented as $k_{t}$.

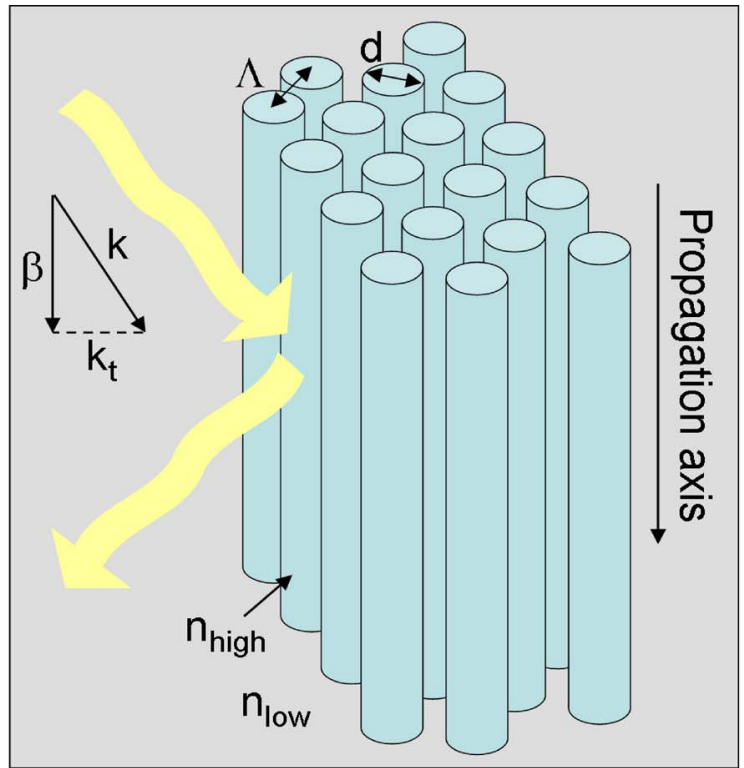

(a)

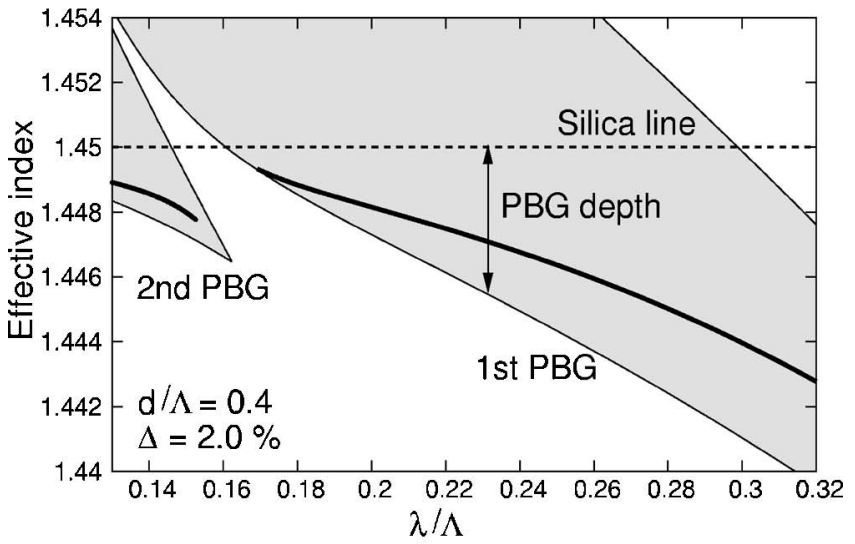

(b)

Fig. 1. (Color online) (a) Schematic representation of light incidence for out-of-plane propagation in a photonic crystal, where high-index rods (refractive index $n_{\text {high }}$ ) with diameter $d$ and pitch $\Lambda$ are structured as triangular lattice in a silica background (refractive index $n_{\text {low }}=1.45$ ). The absolute value of wave vector of $k$ in the material and propagation constant of $\beta$ are assumed for the incident light to the photonic crystal. The transverse component of the wave vector is represented as $k_{t}$. (b) Dispersion curve for the fundamental-like mode calculated by using FEM is depicted for $d / \Lambda=0.4$ and $\Delta=2.0 \%$, where the core is formed by removing one central rod with the cladding of six rings. The shaded region represents the PBG which the photonic crystal cladding exhibits. PBG depth is defined as the effective-index difference between silica and the PBG edge.

In Fig. 1(b), the dispersion curve for the fundamental-like mode calculated by using the FEM [35] is depicted for $d / \Lambda=$ 0.4 and the relative refractive-index difference $\Delta=2.0 \%$, where the core is formed by removing one central rod with the cladding of six rings and $\lambda$ represents the wavelength in free space. The shaded region represents the PBGs formed due to the periodically arranged high-index rods, which is also calculated by using the FEM [34]. The PBG depth is defined as the effective-index difference between silica and the PBG edge. It is well known that in the case of out-of-plane propagation, PBGs are generated sufficiently for the index difference of even less than $1 \%$ [3], and the reason for this can be intuitively understood from the discussion in Ref. [36]. 
According to the concept based on the theory for ARROWs, on the other hand, modes of high-index rods exhibit LP-like profiles at resonant conditions in the photonic crystal, which correspond to the wavelengths at which PBGs disappear. If high-index rods are far enough from the others, the cladding state is not much different from the sum of the modes for each isolated rod. However, when the neighbor rods come close, the modes are affected by them, which leads to the formation of Bloch states. Therefore, the concept of the ARROW theory corresponds to the case for the isolated atom in the wellknown tight-binding theory in solid-state physics [37]. In conclusion, the states satisfy the continuous effective-index values near those for isolated rod modes and the width of the bands is proportional to the overlaps of the isolated rod modes. Accordingly, photonic bands that reside between PBGs are associated with $\mathrm{LP}_{0 m}$ or $\mathrm{LP}_{1 m}$ rod modes because of the large overlap for these modes, while $\mathrm{LP}_{2 m}$ (which nearly coincides with $\mathrm{LP}_{0 m}$ mode), $\mathrm{LP}_{3 m}$, and $\mathrm{LP}_{4 m}$ modes of rods (which occur between $\mathrm{LP}_{0 m}$ and $\mathrm{LP}_{1 m}$ modes) only get across the PBGs without spreading in width, despite the fact that the index-guiding rod modes already satisfy the cutoff condition below the silica line [19], where $m$ is a nonnegative integer. In Fig. 1(b), the band between the first- and second-order PBGs is formed by $\mathrm{LP}_{11}$ rod modes, and it can be seen that the edge that determines the first-order PBG becomes flat by changing the slope below the silica line. In terms of the band theory, this can be understood by the fact that the degenerate $\Gamma, M$, and $K$ states are transformed into the $M$ state absolutely below the silica line, where $\Gamma, M$, and $K$ represent the high symmetry points in the Brillouin zone boundary in reciprocal lattice space for the two-dimensional triangular lattice shown in Fig. 2 (a) and expressed as $\left(k_{x}, k_{y}\right)=(0,0)$ at $\bar{\Gamma}$ point, $\left(k_{x}, k_{y}\right)=(\pi / \Lambda,-\pi / \sqrt{3} \Lambda)$ at $M$ point, and $\left(k_{x}, k_{y}\right)=$ $(4 \pi / 3 \Lambda, 0)$ at $K$ point. In terms of the ARROW theory, on the other hand, it can be explained by the fact that the resonant condition in high-index rods is replaced with that in lowindex regions between the rods below the silica line. This fact means that in order to take into account the resonant feature in the cladding more precisely, consideration of the resonant condition for the low-index material is also required. It is noted that, although the resonant condition in high-index rods is the result of total internal reflection, the other condition is related to antiresonance in rods. However, due to the low localization characteristic of the bound mode supported between the high-index rods, it has not been possible to estimate the PBG edge easily by using the ARROW theory for low-index regions. Here we derive an efficient method based on an ARROW theory, by incorporating a phase relation between the resonant modes in low-index regions for the firstorder PBG.

In. Fig. 2(b), one of the transverse electric field components $\left(E_{x}\right)$ of the Bloch state that determines the floor of the firstorder PBG is depicted for the structure with $d / \Lambda=0.4$ and $\Delta=2.0 \%$. According to the Bloch theorem and ARROW theory, and by analogy to the tight-binding theory, $E_{x}$ concerning the resonant modes in low-index regions must satisfy the following equation $[\underline{37}, \underline{38}]$

$$
E_{x}(\boldsymbol{r}+\boldsymbol{R})=E_{x}(\boldsymbol{r}) \exp \left(i \boldsymbol{k}_{\perp} \cdot \boldsymbol{R}\right)
$$

where $\boldsymbol{r}$ stands for the position vector, $\boldsymbol{R}$ for the translation vector, and $\boldsymbol{k}_{\perp}$ for the in-plane Bloch wave vector correspond-

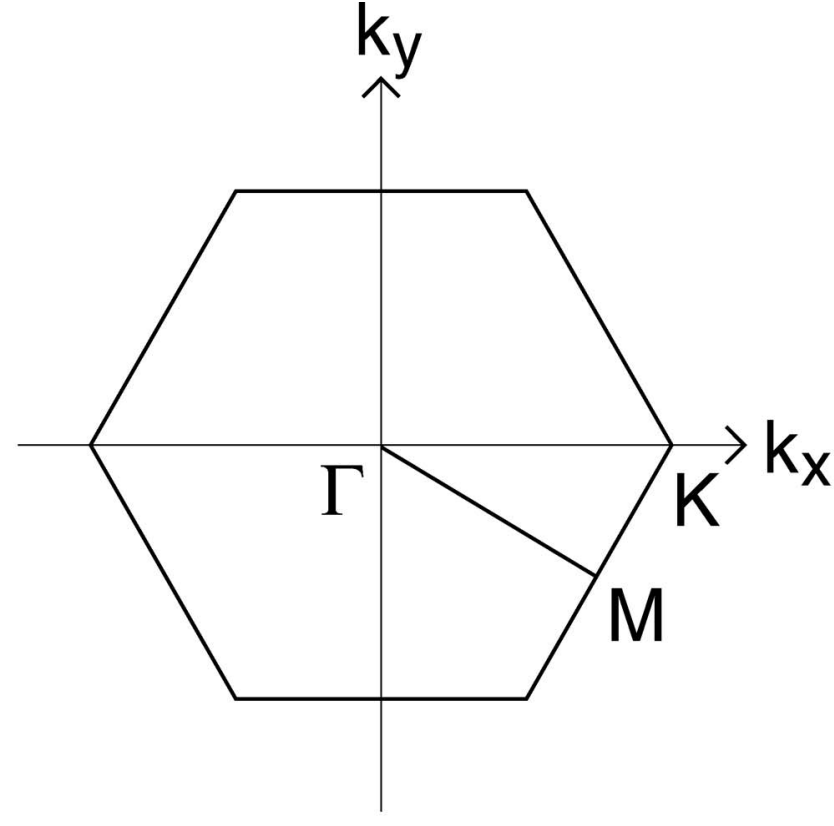

(a)

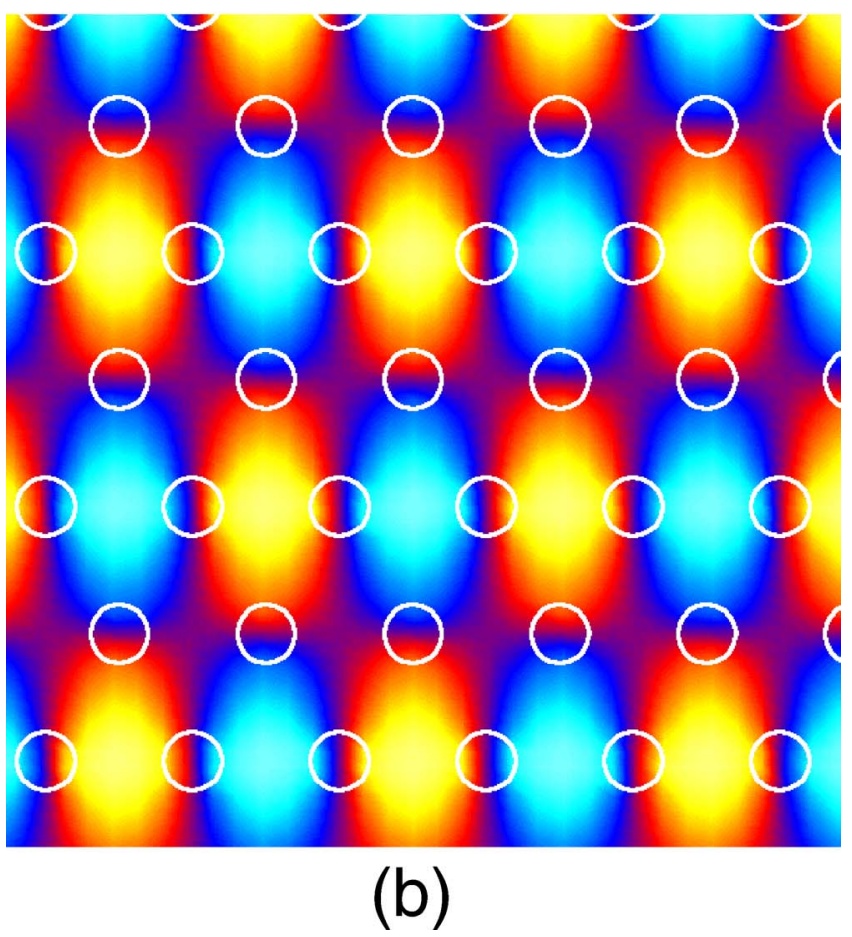

Fig. 2. (Color online) (a) First Brillouin zone boundary in reciprocal lattice space for two-dimensional triangular lattice, where $\left(k_{x}, k_{y}\right)=$ $(0,0)$ at $\Gamma$ point, $\left(k_{x}, k_{y}\right)=(\pi / \Lambda,-\pi / \sqrt{3} \Lambda)$ at $M$ point, and $\left(k_{x}, k_{y}\right)=$ $(4 \pi / 3 \Lambda, 0)$ at $K$ point. (b) One of the transverse electric field components $\left(E_{x}\right)$ of Bloch state which determines the floor of the first-order PBG for the structure with $d / \Lambda=0.4$ and $\Delta=2.0 \%$.

ing to the Brillouin zone boundary. Therefore, a phase shift of one resonant mode from another is related to $\boldsymbol{k}_{\perp} \cdot \boldsymbol{R}$. For example, since the state shown in Fig. 2(b) is related to $M$ point, the phase shift between the nearest neighbor resonant modes is $\pi$. In conventional waveguide couplers with multiple cores, it is known that the effective index for the even state is higher than that for the odd state in general. It is noted that if the state 
shown in Fig. 2(b) is considered as the mode for the ARROWtype coupler, the effective index for the odd state is higher than that for the even one, contrary to the case for conventional waveguides. This is due to the symmetry of the LP mode bound in high-index rods. Because the $\mathrm{LP}_{11}$ rod mode is the antisymmetrical mode in rods, the resultant state for the lowindex regions, which is responsible for the state at the PBG edge, becomes odd when the antisymmetrical mode in rods forms the even state. Here we assume that the Bloch state for the transverse cross section is a two-dimensional standing wave and the transverse wave vector component satisfies $k_{t}=k_{0}\left(n_{\text {low }}^{\prime 2}-n_{\text {edge }}^{2}\right)^{1 / 2}$, where $k_{0}$ is the wavenumber in free space and $n_{\text {edge }}$ represents the effective refractive index of the edge corresponding to the PBG floor. The parameter $n_{\text {low }}^{\prime}$ corresponds to an equivalent refractive index of the cladding material in which high-index inclusions are embedded in the low-index region periodically. Although the material forming the photonic crystal is inhomogeneous, the average equivalent index can be efficiently defined, as given in the next section. It is noted that, according to the state shown in Fig. 2(b), $k_{t}$ must be equal to the absolute value of the fundamental reciprocal lattice vector for the triangular lattice with the pitch of $2 \Lambda$ which the wave forms in transverse direction, because the reciprocal lattice is defined as a set of particular wave vectors composed of plane waves whose periodicity is the same as Bravais lattice, forming a periodic lattice [37]. Therefore, because of the relation $k_{t}=2 \pi / \sqrt{3} \Lambda$, $n_{\text {edge }}$ satisfies the following simple equation:

$$
n_{\text {edge }}=\left(n_{\text {low }}^{2}-\frac{\lambda^{2}}{3 \Lambda^{2}}\right)^{1 / 2}
$$

Here, we take into account the fact that $k \approx n_{\text {low }} \times k_{0}$ (therefore, $n_{\text {low }}^{\prime} \approx n_{\text {low }}$ ) when the values of $d / \Lambda$ and $\Delta$ are small (in particular, $d / \Lambda<0.3$ ), because the cladding material can approximately be considered as the homogeneous silica material and the condition considered is the resonant state in the low-index background between high-index rods. It is worth noting that Eq. (2) does not include any structural parameters of rods ( $d$ and $\Delta$ ) except for $\Lambda$. In contrast to the ARROW theory for high-index rods, where the transmission band in $\lambda$ is largely determined by $d$ and $\Delta$ while not depending on $\Lambda$ [30], this implies that $n_{\text {edge }}$ at $\lambda$ does not depend on $d$ and $\Delta$, but it is largely determined only by $\Lambda$ when $d / \Lambda$ and $\Delta$ are small. In other words, it has a constant value for the structural parameters for which the PBG emerges at the same $\lambda / \Lambda$. In order to confirm this, PBG edges as a function of $\lambda / \Lambda$ calculated by the FEM and Eq. (2) are shown in Fig. 3 for $d / \Lambda=0.207$ and $\Delta=1.48 \%$, and $d / \Lambda=0.255$ and $\Delta=1.0 \%$. The structural parameters are chosen as $d / \Lambda \times$ $\left(n_{\text {high }}^{2}-n_{\text {low }}^{2}\right)^{1 / 2}$, being constant in order to compare the PBG edges emerging at the same $\lambda / \Lambda$, in accordance with the ARROW theory for high-index rods [which can be explained with the aid of Eq. (ㄴ)] [21]. Both of them agree fairly well, and we can see the validity of the proposed formula. Moreover, from Eq. (2), the PBG depth as a function of the structural parameters of the cladding can be derived by replacing $\lambda$ into the structural parameters by using the ARROW theory for high-index rods

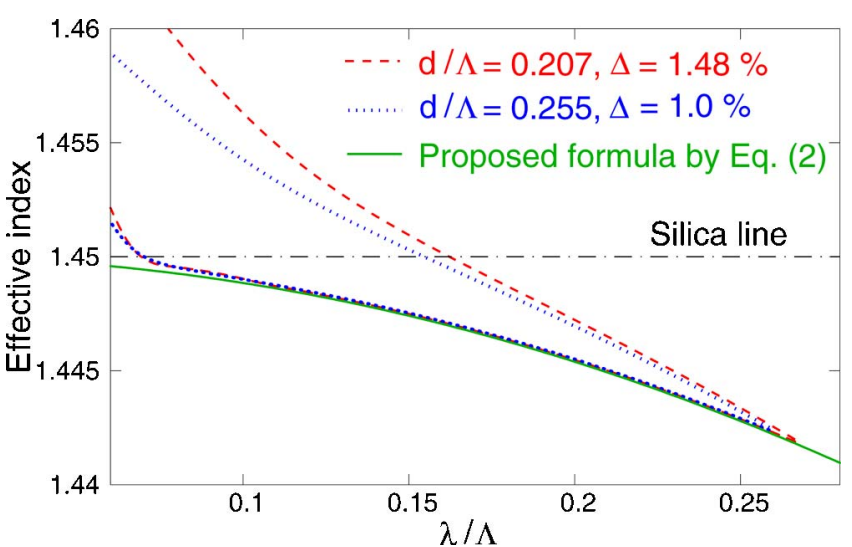

Fig. 3. (Color online) First-order PBG edges as a function of $\lambda / \Lambda$ calculated by FEM (depicted as dashed and dotted curves) and Eq. (2) (depicted as solid curve), where $d / \Lambda=0.207$ and $\Delta=1.48 \%$, and $d / \Lambda=0.255$ and $\Delta=1.0 \%$, respectively.

$$
\text { PBGdepth }=n_{\text {low }}-n_{\text {edge }} \approx\left[\frac{\pi}{\sqrt{3} V}\left(\frac{d}{\Lambda}\right)\right]^{2} n_{\text {low }} \Delta,
$$

where $V$ represents the normalized frequency and is defined for high-index rods as follows:

$$
V=\frac{\pi d}{\lambda}\left(n_{\text {high }}^{2}-n_{\text {low }}^{2}\right)^{1 / 2}=\frac{\pi d}{\lambda} n_{\text {low }}\left(\frac{2 \Delta}{1-2 \Delta}\right)^{1 / 2}
$$

In Eq. (3), since high-index rods are realized by Ge doping in silica for all-solid structures, we assumed that $\Delta$ is small. From the equation derived here, we note that the PBG depth increases monotonically with $\Delta$ and quadratically with $d / \Lambda$.

Recently, it has been reported that high- $\Delta$ all-solid PBGFs can be realized by making use of tellurite glass as the highindex rods [39]. In order to see the validity of the proposed formula for such a case, the effective index of the PBG edges as a function of $\lambda / \Lambda$ calculated by the FEM and Eq. (2) is presented in Fig. 4 for the structural parameters $d / \Lambda=0.17$ and $\Delta=25.7 \%$. Because of the small value of $d / \Lambda$, it is observed that the result obtained by Eq. (2) clearly agrees well with that by the FEM.

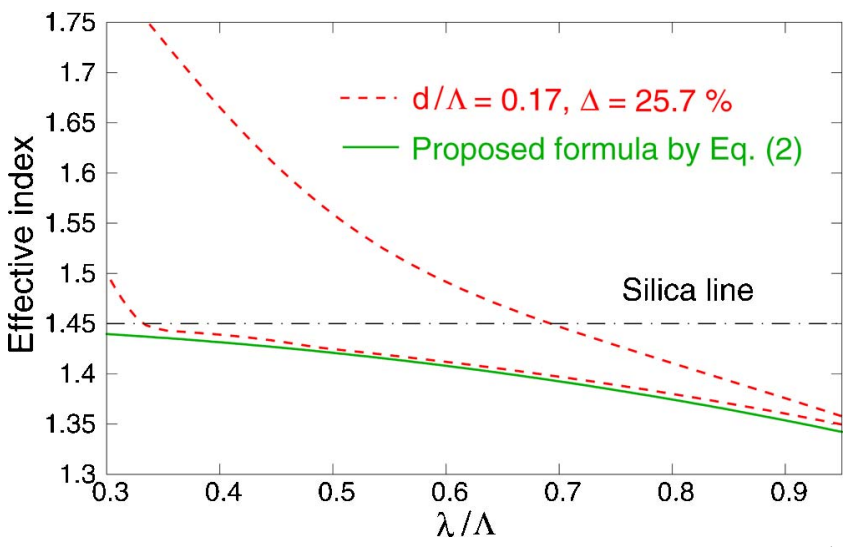

Fig. 4. (Color online) First-order PBG edges as a function of $\lambda / \Lambda$ calculated by FEM (depicted as dashed curve) and Eq. (2) (depicted as solid curve), where $d / \Lambda=0.17$ and $\Delta=25.7 \%$ [39]. 


\section{SPECIFYING FACTORS OF REDUCING PHOTONIC BANDGAP DEPTH}

In the previous section, we assumed that high-index inclusions embedded in the low-index material do not have an effect on the equivalent refractive index of the material when the value of $d / \Lambda$ is small because the resonant condition in the low-index background was considered. When the value of $d / \Lambda$ is increased $(d / \Lambda \geq 0.3)$, however, the effect of highindex rods on the resonant modes in low-index regions is inevitable due to the fact that each high-index rod approaches the center of the low-index region in this case. As discussed below, because the effect is one of the factors for increasing the value of $n_{\text {edge }}$ (accordingly reducing the PBG depth) with increasing $d / \Lambda$, the theory provided in Sect. 2 should be revised slightly in order to incorporate the impact of high-index rods on the equivalent refractive index of the material. In Fig. 5, we show the normalized wavelength dependence of PBG edges for $d / \Lambda=0.4$ and $\Delta=2.0 \%$ calculated by the FEM and the predicted result by Eq. (2). The actual edge corresponding to the PBG floor calculated by the FEM shifts upward when compared to the prediction presented in Eq. (2), where the effect of high-index rods on the equivalent refractive index of the material is ignored. This indicates that the approximation $n_{\text {low }}^{\prime} \approx n_{\text {low }}$ applied above is not appropriate for structures with large $d / \Lambda$ and, more precisely, $n_{\text {low }}^{\prime}$ should be given as the square root of the average relative permittivity weighted by the electric field intensity $\left(|\boldsymbol{E}|^{2}\right)$ as follows:

$$
n_{\text {low }}^{\prime 2}=\sum_{i=\text { low,high }} \frac{\int_{S_{i}} n_{i}^{2}|\boldsymbol{E}|^{2} \mathrm{~d} S}{\int_{S}|\boldsymbol{E}|^{2} \mathrm{~d} S}
$$

Although this concept is useful when the length scale of the variation of the field is larger as compared to that of the inhomogeneities, it would explain the phenomenon approximately in our system. Because the refractive indices are constant in each homogeneous material, the formula in Eq. (ㄷ) can be expressed as

$$
n_{\mathrm{low}}^{\prime}=n_{\mathrm{low}}\left\{1+I\left(\frac{2 \Delta}{1-2 \Delta}\right)\right\}^{1 / 2},
$$

where $I$ is the modal overlap integral in the high-index rods normalized by that in the cross section

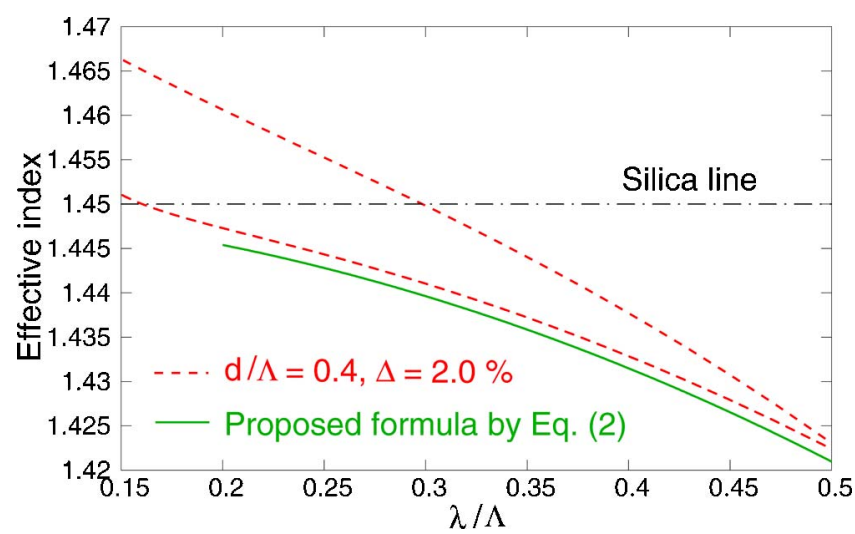

Fig. 5. (Color online) First-order PBG edges as a function of $\lambda / \Lambda$ calculated by FEM (depicted as dashed curve) and Eq. (2) (depicted as solid curve) for the case that $d / \Lambda$ is not small, where $d / \Lambda=0.4$ and $\Delta=2.0 \%$. The impact of high-index rods is clearly seen.

$$
I=\frac{\int_{S_{\text {high }}|\boldsymbol{E}|^{2} \mathrm{~d} S}}{\int_{S}|\boldsymbol{E}|^{2} \mathrm{~d} S} .
$$

According to the discussion above, the enlargement of the equivalent refractive index of the cladding material for the Bloch state is due to the penetration of light for the resonant modes bound in low-index regions into high-index rods. Therefore, the effective index of the edge corresponding to the PBG floor is given by the following equation for any structural parameters:

$$
n_{\text {edge }}=\left[n_{\text {low }}^{\prime 2}-\frac{\lambda^{2}}{3 \Lambda^{2}}\right]^{1 / 2} .
$$

Here, we approximate the Bloch state corresponding to $M$ point (the state shown in Fig. 4b of Ref. [38] or Fig. 2(b) shown above) by the simple cosine function. In this case, the value of $I$ can be obtained efficiently:

$$
\begin{aligned}
I \approx & \frac{\pi}{2 \sqrt{3}}(d / \Lambda)^{2}\left\{1+\frac{2}{3 \pi} \cos \left(\frac{2 \pi}{\sqrt{3}}(d / \Lambda)\right)\right\} \\
& -\frac{1}{\pi}(d / \Lambda) \sin \left(\frac{2 \pi}{\sqrt{3}}(d / \Lambda)\right) .
\end{aligned}
$$

In Fig. 6, we show the normalized wavelength dependence of PBG edges calculated by the FEM and the results obtained by Eq. (8), for $d / \Lambda=0.4$ and $\Delta=2.0 \%$ in (a), $d / \Lambda=0.5$ and $\Delta=3.0 \%$ in (b), and $d / \Lambda=0.6$ and $\Delta=2.0 \%$ in (c). We can clearly see the validity of the proposed formula, taking into account the factor associated with the penetration of light into rods. We note that a small amount of the discrepancy of the result from that obtained by the FEM is due to the approximation applied to obtain $I$ by using Eq. (9), where the Bloch state was assumed to be the simple cosine function. In fact, we confirmed that the result agrees fairly well with the FEM result if the value of $I$ in Eq. (7) is calculated by using the modal solver.

Once the equation for the effective index of the PBG edge as a function of wavelength is derived, it is possible to derive the relationship of the PBG edge or PBG depth between any actual structural parameters by applying the ARROW theory for high-index rods. By substituting Eq. (ㄸ) into Eq. (ㅁ) $)$, the following relation can be obtained:

$$
n_{\text {edge }}=\left[n_{\text {low }}^{\prime 2}-\frac{1}{3}\left\{\frac{\pi}{V}\left(\frac{d}{\Lambda}\right) n_{\text {low }}\right\}^{2}\left(\frac{2 \Delta}{1-2 \Delta}\right)\right]^{1 / 2} .
$$

According to the ARROW theory for high-index rods expressed in Eq. (므), the condition that $V=1.6$ is sufficiently included in the first-order PBG in frequency, because the edge of the PBG for the highest frequency is $V \approx 2.4$, corresponding to the cut-off frequency of $\mathrm{LP}_{11}$ mode in conventional fibers. In order to see the relationship of the PBG depth between actual structural parameters, we show the PBG depth $\left(=n_{\text {low }}-n_{\text {edge }}\right)$ as a function of $\Delta$ for $d / \Lambda=0.2,0.3$, and 0.4 , and as a function of $d / \Lambda$ for $\Delta=0.8 \%$ and $2.0 \%$ in Figs. 7(a) and 7(b), respectively, where $V$ is fixed as 1.6. As a reference, the results obtained by using the FEM are also depicted. We can see the quadratic increase of the PBG depth with increasing $d / \Lambda$ for each $\Delta$ when $d / \Lambda$ is small, as well as the monotonic increase with increasing $\Delta$ for each $d / \Lambda$. In Fig. $7(\mathrm{~b})$, 


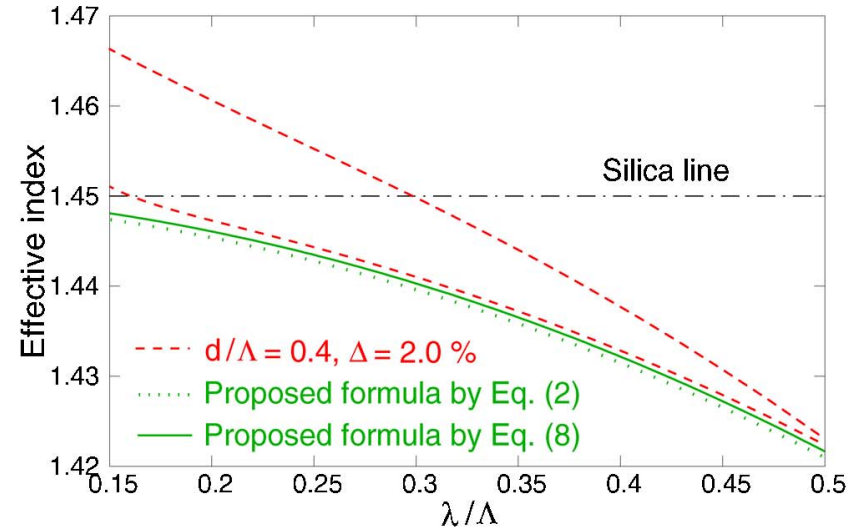

(a)

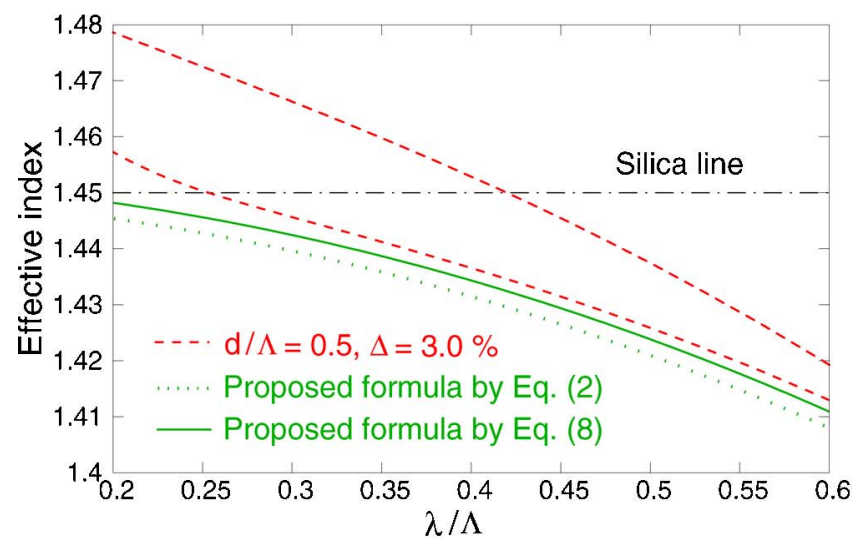

(b)

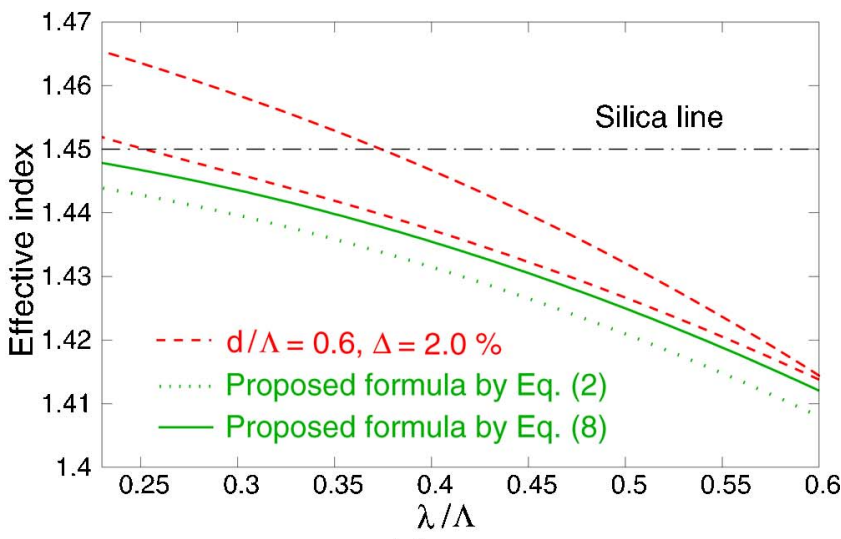

(c)

Fig. 6. (Color online) First-order PBG edges as a function of $\lambda / \Lambda$ calculated by FEM (depicted as dashed curve) and Eq. (8) taking into account the effect of the high-index rods on the wavenumber in the cladding material (depicted as solid curve) for the case that $d / \Lambda$ is not small, where (a) $d / \Lambda=0.4$ and $\Delta=2.0 \%$, (b) $d / \Lambda=0.5$ and $\Delta=3.0 \%$, and (c) $d / \Lambda=0.6$ and $\Delta=2.0 \%$. As a reference, the result obtained by Eq. (2) is also shown in which the refractive index of the cladding material is assumed to be $n_{\text {low }}$ (depicted as dotted curve).

when $d / \Lambda$ is increased, the slope of the PBG depth decreases. This means that the enlargement in the effect of high-index rods on the wavenumber in the cladding material is one of the factors for increasing the value of $n_{\text {edge }}$ (accordingly reducing the PBG depth) with increasing $d / \Lambda$. It is worth noting that this effect is interpreted as penetration of a small amount of light for the resonant modes bound in low-index regions into high-index rods. In Figs. 8(a) and 8(b), we also show the PBG depth as a function of $\Delta$ for $\overline{d / \Lambda}=0.2,0.3$, and

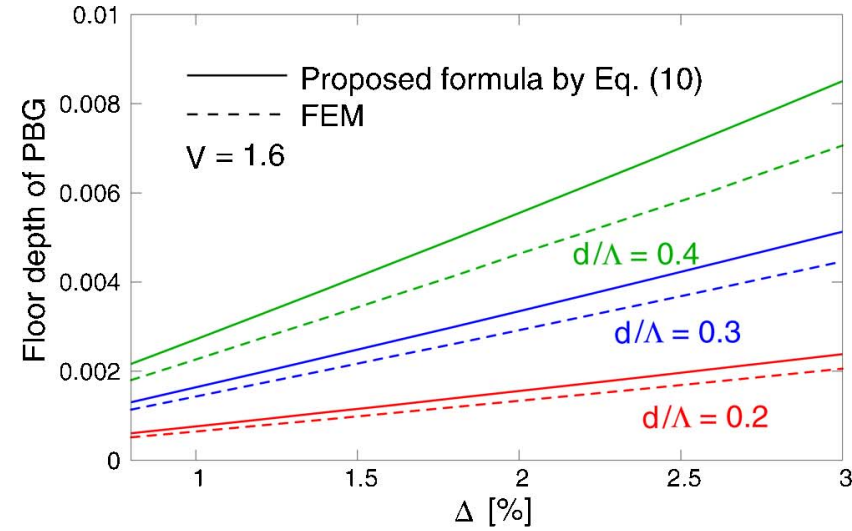

(a)

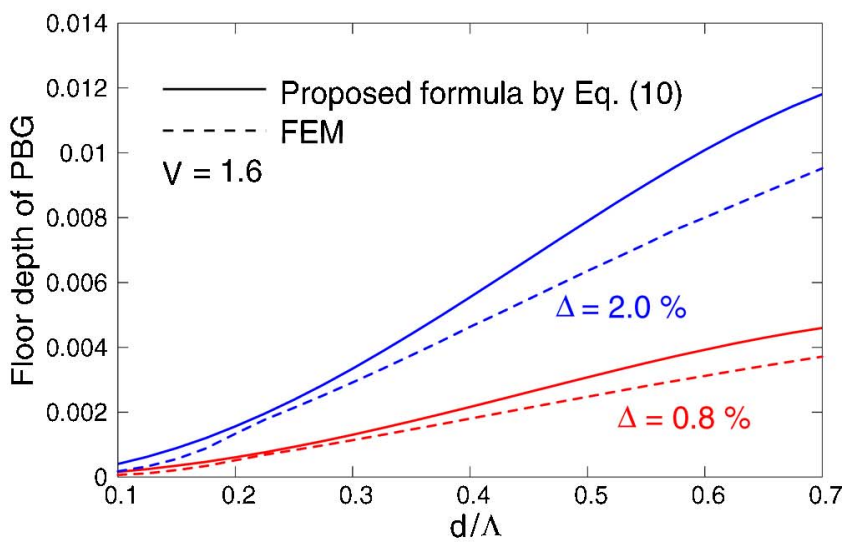

(b)

Fig. 7. (Color online) First-order PBG depth as a function of structural parameters of (a) $\Delta$ and (b) $d / \Lambda$; where $V$ is fixed as 1.6. In the figures, the dashed curves are the results obtained by FEM and the solid curves are those obtained by Eq. (10) taking into account the effect of the high-index rods on the wavenumber in the cladding material. The value of $d / \Lambda$ is fixed as $0.2,0.3$, and 0.4 for each curve in (a), and the value of $\Delta$ is fixed as $0.8 \%$ and $2.0 \%$ for each curve in (b).

0.4 , and that as a function of $d / \Lambda$ for $\Delta=0.8 \%$ and $2.0 \%$ at the midgap wavelength, for the results by the FEM and the proposed formula by Eq. (10). In order to derive the value of $V$ at the midgap wavelength for the results by the proposed formula, because it should not be acquired by using numerical simulations, we used the analytical formula obtained by the following expressions [33]

$$
\begin{gathered}
J_{0}\left(V_{01}\right)+\frac{1}{2} V_{01} J_{1}\left(V_{01}\right) \ln f=0, \\
J_{0}\left(V_{11}\right)-J_{2}\left(V_{11}\right) f=0,
\end{gathered}
$$

where $J_{0}, J_{1}$, and $J_{2}$ are Bessel functions, $V_{01}$ is the value of $V$ corresponding to the lowest frequency for the first-order $\mathrm{PBG}$ on the silica line determined by the $\mathrm{LP}_{01}$ rod modes, $V_{11}$ is the value corresponding to the highest frequency determined by the $\mathrm{LP}_{11}$ rod modes, and $f$ is the filling fraction of high-index rods in the low-index background for the triangular lattice structure defined in Ref. [33]. According to Eqs. (11) and (12), the value of $V_{c}$ that corresponds to the midgap wavelength is derived as follows:

$$
V_{c}=\frac{2 V_{01} V_{11}}{V_{01}+V_{11}} .
$$




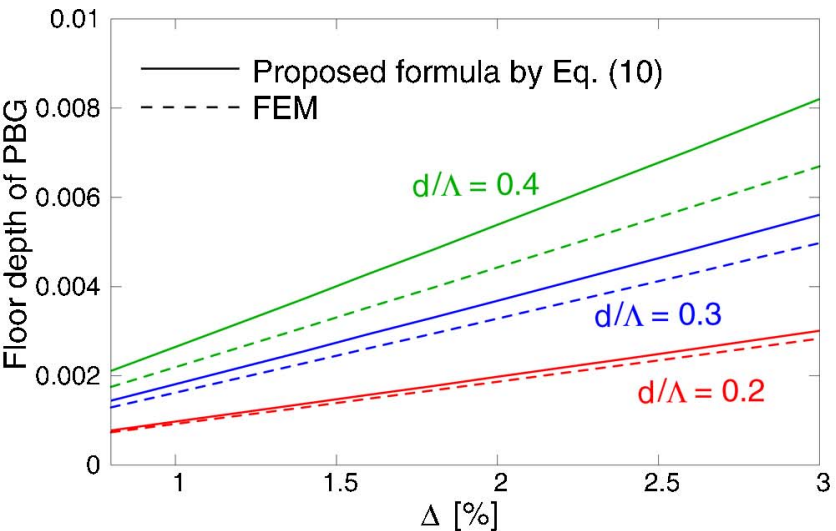

(a)

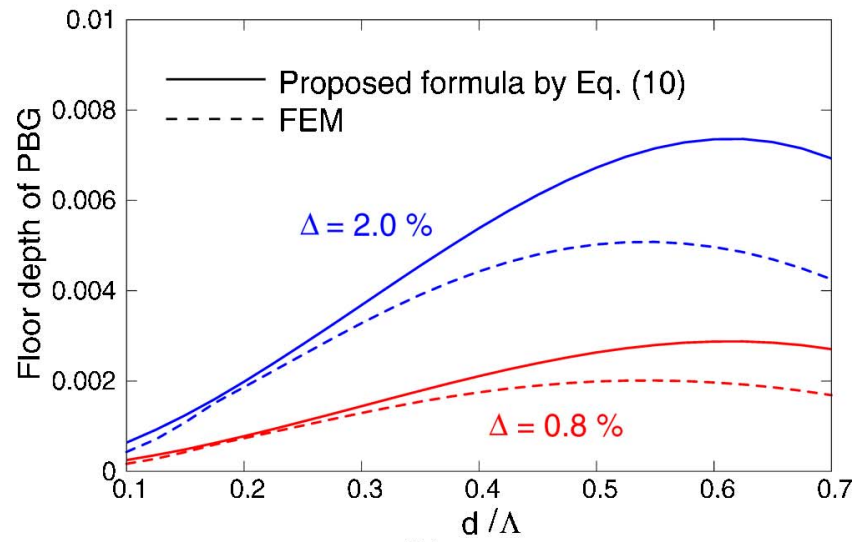

(b)

Fig. 8. (Color online) First-order PBG depth as a function of structural parameters of (a) $\Delta$ and (b) $d / \Lambda$; where $V$ value is given for the central wavelength of the PBG for each structure. In the figures, the dashed curves are the results obtained by FEM and the solid curves are those obtained by Eq. (10) taking into account the effect of the high-index rods on the wavenumber in the cladding material. The value of $d / \Lambda$ is fixed as $0.2,0.3$, and 0.4 in (a), and the value of $\Delta$ is fixed as $0.8 \%$ and $2.0 \%$ in (b). A certain percentage for the deviation for each structural parameter is due to the discrepancy of the given values of $V_{c}$.

Although the actual $V_{c}$ is not in accordance with the value obtained by Eq. (13) in a precise sense, it is found that a qualitative agreement can be obtained between the formula and the FEM results. In Fig. 8(b), when the exploitation of the midgap wavelength is considered, not only does the slope of the PBG edge decrease, but the value itself is also reduced for increasing $d / \Lambda$. This is because, due to the blue shift of $V_{01}$ for the structures with a large value of $d / \Lambda$, the reduction of PBG depth caused by the effect of high-index rods explained above is strongly incurred at the midgap wavelength. Again, it is noted that the discrepancy of the results in Figs. 7 and 8 is due to the approximation applied to obtain $I$ by using Eq. (9), as mentioned above. Moreover, we also note that the values obtained by using the FEM are always slightly smaller than those from the proposed formula in Figs. $\underline{7}$ and $\underline{8}$, which occurs even if the discrepancy induced by the approximation is ignored. By considering the deviation in the results, the other factors of reducing the PBG depth (increasing $n_{\text {edge }}$ ) are successfully identified, although a small amount of the deviation in Fig. 8 is due to the discrepancy of given $V_{c}$ between the FEM and the approximation calculated by Eq. (13). In particular, for the structures with a larger value of $d / \bar{\Lambda}$, the deviation becomes larger. The reason for this is related to the fact that the width of the photonic band formed by the $\mathrm{LP}_{11}$ rod modes increases when $d / \Lambda$ has a large value. If we look at Fig. 6 again, although the edge provided by the proposed formula agrees to some extent with that by the FEM over the entire wavelength range, the result obtained by the proposed formula deviates from that calculated by the FEM slightly at the condition of changing the slope of the edge (near the blue edge of the PBG) which is explained in the previous section. It is also found that another factor concerning the reduction of the PBG depth is operating when $d / \Lambda$ has a small value $(d / \Lambda \approx 0.1)$. Although finding the physics underlying this observation is challenging, at this time we have found that it is related to the effect of the formation of the edge for the second-order PBG on the first-order one. We are now in the process of investigating the cause and the impact on the fiber properties. The detailed discussion will be presented elsewhere.

Finally, we would like to comment on the application of the theory to the other PBGs. Although the Bloch state at the edge corresponding to the PBG floor and, therefore, $k_{t}$, is different for each PBG, an intuitive understanding can be obtained by the discussion provided here. In particular, there is a room for considering the reason for the formation of the shallow PBG for the even-order ones; in Ref. [20], it was shown to be due to the differing impact of the modal overlap for the LP modes bound in high-index rods between the even- and odd-order PBGs. However, because the edge concerning the PBG floor is related to resonance in low-index regions [33], according to the theory provided here, another reason must also exist for this, in addition to that shown in [20]. Figure 9 shows the Bloch state at the edge corresponding to the floor of the second-order PBG at the midgap wavelength for $d / \Lambda=0.2$ and $\Delta=1.0 \%$. Because the state is related to $\Gamma$ point shown in Fig. 2(a), the phase shift between the nearest neighbor resonant modes is 0 . Again, from the point of view of the ARROW-

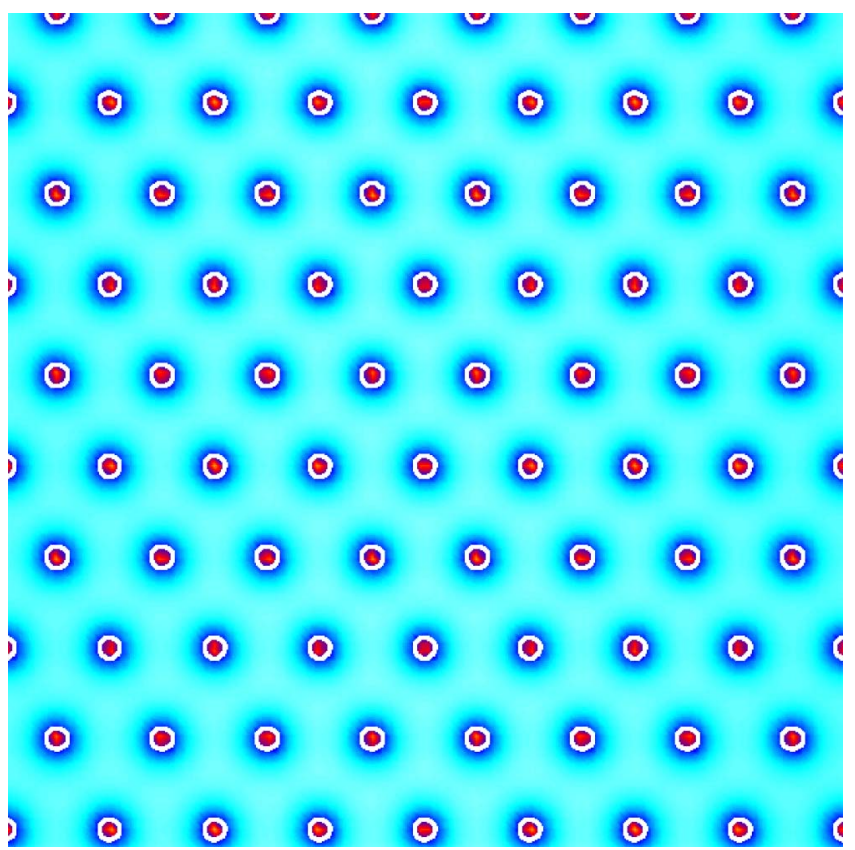

Fig. 9. (Color online) One of the transverse electric field components $\left(E_{x}\right)$ of Bloch state that determines the floor of the second-order PBG for the structure with $d / \Lambda=0.2$ and $\Delta=1.0 \%$ at the midgap wavelength. The state is formed with the condition of $\Gamma$ point shown in Fig. 2(a). The value of $n_{\text {edge }}=1.449916$, which is very close to the refractive index of silica $\left(n_{\text {low }}=1.45\right)$. 
type coupler, because the band which determines the edge is $\mathrm{LP}_{02}$ mode (symmetrical mode) bound in high-index rods, the effective index for the even state confined in low-index regions is higher than that for the odd state. According to an analogy with Eq. (8), when the effect of high-index rods on the wavenumber in the cladding material is small (in other words, the value of $d / \Lambda$ and $\Delta$ is small) and, therefore, $n_{\text {low }}^{\prime} \approx n_{\text {low }}$, the value of $n_{\text {edge }}$ would show an asymptotic convergence to a nearly equal value of $n_{\text {low }}$ for the second-order PBG. This is because; as deduced from Fig. 9 where the material along which light propagates can be considered to be homogeneous for the transverse direction with the refractive index of $n_{\text {low }}$ and accordingly $k \approx n_{\text {low }} \times k_{0} \approx \beta$, the factor responsible for decreasing the value of $n_{\text {edge }}$ in Eq. (8) or Eq. (2), which has a relation with $k_{t} / k_{0}$, must be smaller as compared to the case in the firstorder PBG. In fact, the value of $n_{\text {edge }}$ at the state shown in Fig. 9 (the case that the effect of high-index rods is small) is about 1.449916, which is very close to the refractive index of silica $\left(n_{\text {low }}=1.45\right)$. Moreover, a part of this effect can also be seen in Figs. $7(\mathrm{~b})$ and $8(\mathrm{~b})$ at $d / \Lambda \approx 0.1$ for the result by using the FEM, where the actual edge of the first-order PBG is determined by the condition of the second-order PBG as mentioned above. It is observed that the depth shown by the dashed curves clearly goes to 0 for the structure with a small value of $d / \Lambda$ in the figures. Even when the effect of high-index rods increases and, therefore, the value of $k_{0}$ at which the PBG emerges decreases, however, $k_{t} / k_{0}$ itself would still have a small value. In conclusion, because the LP modes bound in high-index rods are symmetrical for the even-order PBGs, the factor responsible for decreasing the value of $n_{\text {edge }}$ or increasing the PBG depth becomes smaller when compared to the case in the odd-order PBGs, where the LP rod modes are antisymmetrical.

\section{CONCLUSIONS}

In this paper, we proposed a simple formula that gives the effective refractive index of the first-order PBG edge for the maximum propagation angle, which corresponds to the PBG floor in all-solid PBGFs, based on an ARROW theory incorporating a phase relation between the resonant modes in low-index regions. We have shown that the effective index of the PBG edge has a constant value for the structural parameters with which the PBG emerges at the same wavelength normalized by the pitch when normalized rod diameters are small. By incorporating the ARROW theory for high-index rods, it has been theoretically shown that the PBG depth exhibits a monotonic increase with the relative refractive index difference for rods and quadratic increase with the rod diameter normalized by the pitch. In addition, the cause of the reduction of the PBG depth with increasing the rod diameter normalized by the pitch and the refractive-index difference has been successfully found. We have shown that the change of the wavenumber for the resonant modes bound in lowindex regions due to the small amount of penetration of the light into high-index rods is the critical factor that is responsible for the reduction of the PBG depth. Moreover, it has been shown that the effect of the enlargement of the photonic-band width due to the modal overlap of rod modes is also the major factor for reducing the PBG depth. The validity of the proposed simple formula, which can be applied for any structural parameters, has been verified by using the vectorial solver based on the FEM. Finally, the critical reason for decreasing the PBG depth in even-order PBGs has been discussed based on the theory provided here.

\section{ACKNOWLEDGMENTS}

The authors would like to kindly acknowledge the financial support of this project from the Japan Society for the Promotion of Science (JSPS), Grant-in-Aid for JSPS Fellows (20-2266). They are thankful to Dr. S. Roy from Hokkaido University for stimulating discussions.

\section{REFERENCES}

1. J. C. Knight, F. Luan, G. J. Pearce, A. Wang, T. A. Birks, and D. M Bird, "Solid photonic bandgap fibres and applications," Jpn. J. Appl. Phys. 45, 6059-6063 (2006).

2. F. Luan, A. K. George, T. D. Hedley, G. J. Pearce, D. M. Bird, J. C. Knight, and P. St. J. Russell, "All-solid photonic bandgap fiber," Opt. Lett. 29, 2369-2371 (2004).

3. A. Argyros, T. A. Birks, S. G. Leon-Saval, C. M. B. Cordeiro, F. Luan, and P. St. J. Russell, "Photonic bandgap with an index step of one percent," Opt. Express 13, 309-314 (2005).

4. A. Argyros, T. A. Birks, S. G. Leon-Saval, C. M. B. Cordeiro, and P. St. J. Russell, "Guidance properties of low-contrast photonic bandgap fibres,” Opt. Express 13, 2503-2511 (2005).

5. G. Bouwmans, L. Bigot, Y. Quiquempois, F. Lopez, L. Provino, and M. Douay, "Fabrication and characterization of an all-solid $2 \mathrm{D}$ photonic bandgap fiber with a low-loss region $(<20 \mathrm{~dB} / \mathrm{km})$ around 1550 nm,” Opt. Express 13, 8452-8459 (2005).

6. A. Wang, A. K. George, and J. C. Knight, "Three-level neodymium fiber laser incorporating photonic bandgap fiber," Opt. Lett. 31, 1388-1390 (2006).

7. J. M. Stone, G. J. Pearce, F. Luan, T. A. Birks, J. C. Knight, A. K. George, and D. M. Bird, "An improved photonic bandgap fiber based on an array of rings," Opt. Express 14, 6291-6296 (2006).

8. A. Bétourné, V. Pureur, G. Bouwmans, Y. Quiquempois, L. Bigot, M. Perrin, and M. Douay, "Solid photonic bandgap fiber assisted by an extra air-clad structure for low-loss operation around 1.5 m ," Opt. Express 15, 316-324 (2007).

9. A. Bétourné, G. Bouwmans, Y. Quiquempois, M. Perrin, and M. Douay, "Improvements of solid-core photonic bandgap fibers by means of interstitial air holes," Opt. Lett. 32, 1719-1721 (2007).

10. V. Pureur, L. Bigot, G. Bouwmans, Y. Quiquempois, M. Douay and Y. Jaouen, "Ytterbium-doped solid core photonic bandgap fiber for laser operation around $980 \mathrm{~nm}$," Appl. Phys. Lett. 92, 061113 (2008).

11. C. B. Olausson, C. I. Falk, J. K. Lyngs $\varphi$, B. B. Jensen, K. T. Therkildsen, J. W. Thomsen, K. P. Hansen, A. Bjarklev, and J. Broeng, "Amplification and ASE suppression in a polarization-maintaining ytterbium-doped all-solid photonic bandgap fibre," Opt. Express 16, 13657-13662 (2008).

12. A. Shirakawa, H. Maruyama, K. Ueda, C. B. Olausson, J. K. Lyngs $\varnothing$, and J. Broeng, "High-power Yb-doped photonic bandgap fiber amplifier at 1150-1200 nm," Opt. Express 17, 447-454 (2009).

13. V. Pureur, J. C. Knight, and B. T. Kuhlmey, "Higher order guided mode propagation in solid-core photonic bandgap fibers," Opt. Express 18, 8906-8915 (2010).

14. T. T. Larsen, A. Bjarklev, D. S. Hermann, and J. Broeng, "Optical devices based on liquid crystal photonic bandgap fibres," Opt. Express 11, 2589-2596 (2003).

15. N. M. Litchinitser, S. C. Dunn, P. E. Steinvurzel, B. J. Eggleton, T. P. White, R. C. McPhedran, and C. M. de Sterke, "Application of an ARROW model for designing tunable photonic devices," Opt. Express 12, 1540-1550 (2004).

16. T. T. Alkeskjold, J. Lægsgaard, A. Bjarklev, D. S. Hermann, A. Anwatti, J. Broeng, J. Li, and S. Wu, "All-optical modulation in dye-doped nematic liquid crystal photonic bandgap fibers," Opt. Express 12, 5857-5871 (2004).

17. M. W. Haakestad, T. T. Alkeskjold, M. D. Nielsen, L. Scolari, J. Riishede, H. E. Engan, and A. Bjarklev, "Electrically tunable photonic bandgap guidance in a liquid-crystal-filled photonic crystal fiber," IEEE Photonics Technol. Lett. 17, 819-821 (2005). 
18. T. T. Alkeskjold and A. Bjarklev, "Electrically controlled broadband liquid crystal photonic bandgap fiber polarimeter," Opt. Lett. 32, 1707-1709 (2007).

19. J. Lægsgaard, "Gap formation and guided modes in photonic bandgap fibres with high-index rods," J. Opt. A Pure Appl. Opt. 6, 798-804 (2004).

20. T. A. Birks, F. Luan, G. J. Pearce, A. Wang, J. C. Knight, and D. M. Bird, "Bend loss in all-solid bandgap fibres," Opt. Express 14, 5688-5698 (2006).

21. T. Murao, K. Saitoh, and M. Koshiba, "Detailed theoretical investigation of bending properties in solid-core photonic bandgap fibers," Opt. Express 17, 7615-7629 (2009)

22. T. Murao, K. Saitoh, T. Taru, T. Nagashima, K. Maeda, T. Sasaki, and M. Koshiba, "Bend-insensitive and effectively single-moded all-solid photonic bandgap fibers with heterostructured cladding," in Proceedings of European Conference on Optical Communication (ECOC 2009), paper 2.1.4.

23. A. A. Maradudin and A. R. McGurn, "Out of plane propagation of electromagnetic waves in a two-dimensional periodic dielectric medium,” J. Mod. Opt. 41, 275-284 (1994).

24. M. J. F. Digonnet, H. K. Kim, G. S. Kino, and S. Fan, "Understanding air-core photonic-bandgap fibers: analogy to conventional fibers," J. Lightwave Technol. 23, 4169-4177 (2005).

25. Y. Li, C. Wang, T. A. Birks, and D. M. Bird, "Effective index method for all-solid photonic bandgap fibres," J. Opt. A Pure Appl. Opt. 9, 858-861 (2007).

26. Y. Li, D. M. Bird, and T. A. Birks, "Bend loss in all-solid bandgap fibers revisited,” J. Lightwave Technol. 28, 1368-1372 (2010).

27. T. Murao, K. Saitoh, K. Nagao, and M. Koshiba, "Design principle for low bending losses in all-solid photonic bandgap fibers," in Proceedings of Conference on Lasers and Electro-Optics and Quantum Electronics and Laser Science Conference (CLEO/ QELS 2010), paper JTuD45.

28. M. A. Duguay, Y. Kokubun, T. L. Koch, and L. Pfeiffer, "Antiresonant reflecting optical waveguides in $\mathrm{SiO}_{2}-\mathrm{Si}$ multilayer structures," Appl. Phys. Lett. 49, 13-15 (1986).
29. T. Baba and Y. Kokubun, "Dispersion and radiation loss characteristics of antiresonant reflecting optical waveguidesnumerical results and analytical expressions," IEEE J. Quantum Electron. 28, 1689-1700 (1992).

30. N. M. Litchinitser, S. C. Dunn, B. Usner, B. J. Eggleton, T. P. White, R. C. McPhedran, and C. M. de Sterke, "Resonances in microstructured optical waveguides," Opt. Express 11, 1243-1251 (2003).

31. P. Steinvurzel, B. T. Kuhlmey, T. P. White, M. J. Steel, C. M. de Sterke, and B. J. Eggleton, "Long wavelength anti-resonant guidance in high index inclusion microstructured fibers," Opt. Express 12, 5424-5433 (2004).

32. K. J. Rowland, S. V. Afshar, and T. M. Monro, "Bandgaps and antiresonances in integrated-ARROWs and Bragg fibers; a simple model," Opt. Express 16, 17935-17951 (2008).

33. T. A. Birks, G. J. Pearce, and D. M. Bird, "Approximate band structure calculation for photonic bandgap fibres," Opt. Express 14, 9483-9490 (2006).

34. T. Murao, K. Saitoh, and M. Koshiba, "Design of air-guiding modified honeycomb photonic band-gap fibers for effectively single-mode operation," Opt. Express 14, 2404-2412 (2006).

35. K. Saitoh and M. Koshiba, "Full-vectorial imaginary-distance beam propagation method based on a finite element scheme: application to photonic crystal fibers," IEEE J. Quantum Electron. 38, 927-933 (2002).

36. T. A. Birks, D. M. Bird, T. D. Hedley, J. M. Pottage, and P. St. J. Russell, "Scaling laws and vector effects in bandgap-guiding fibres," Opt. Express 12, 69-74 (2004).

37. N. W. Ashcroft and N. D. Mermin, Solid State Physics (Holt, Rinehart, and Winston, 1976).

38. M. Perrin, Y. Quiquempois, G. Bouwmans, and M. Douay, "Coexistence of total internal reflection and bandgap modes in solid core photonic bandgap fibre with interstitial air holes," Opt. Express 15, 13783-13795 (2007).

39. M. A. Schmidt, N. Granzow, N. Da, M. Peng, L. Wondraczek, and P. St. J. Russell, "All-solid bandgap guiding in tellurite-filled silica photonic crystal fibers," Opt. Lett. 34, 1946-1948 (2009). 